\title{
CAUSES OF FILM PROPERTY REPRODUCIBILITY PROBLEM IN MAGNETRON SPUTTERING
}

\author{
Mwabora, J. M+. and R. T. Kivaisi* \\ +Department of Physics, University of Nairobi \\ P.O.Box 30197, Nairobi, Kenya \\ *Department of Physics, University of Dar es Salaam \\ Dar es Salaan, Tanzania \\ E-mail: mwabora@uonbi.ac.ke
}

\begin{abstract}
Causes of reproducibility problem in thin film deposition using magnetron sputtering have been identified. To remedy this problem, one must calibrate deposition rate after installation of a new target or magnetron and should repeat such calibrations as frequently as possible in order to obtain films with reproducible properties. For magnetrons used in our laboratory to be in working condition, the transverse magnetic field strength on the surface of the magnetron should be about $50 \mathrm{mT}$ or more.
\end{abstract}

Key words: Thin films, Magnetron sputtering, Tranverse magnetic field strength, $\mathrm{Cu}$ films, In films, Deposition rate.

\section{INTRODUCTION}

It is only through experience that one comes to find out that working with sputtering systems is not as straight forward as it appears in the operation manual. For example, our experience with magnetron sputtering system has associated reproducibility problem with damaging of magnetrons and changes in deposition rate with prolonged use of a magnetron.

Damaging of the magnetrons is mainly caused by lack of cooling water and in extreme cases electrical shorting of the cathode. The resistance of the cooling water must be high to assure proper operation of the magnetron. With the cooling water flowing in the magnetron, the resistance from the target (cathode surface if metallic) to anode (ground shield) must exceed $3 \mathrm{M} \Omega$ for DC or $5 \mathrm{M} \Omega$ for RF operation, otherwise power loss will occur at the cathode due to low impedance provided by the cooling water to earth [US Patent No. 4,434,042, 1992] Electrical shorting of the cathode results in high temperature causing severe damage to target material, cathode surface and the magnetrons. Higher temperatures for the heater element in the deposition chamber can also damage the magnetrons by weakening the magnetic field strength.

Magnetic field strengths of about $50 \mathrm{mT}$ parallel to the target surface combined with the electric field in magnetrons cause the secondary electrons to drift in a closed circuit or "magnetron tunnel" in front of the target surface [Vossen and Kern, 1991; Thornton, 1978; Thornton and Penfold, 1978; Thornton, 1979]. Both the North-South-
North (N-S-N) and South-North-South (S-N-S) configuration for magnets in the magnetrons are commercially available and described in the literature [Vossen and Kern, 1991]; Chapman, 1980]. Chapman, (1980) gave the configuration for a square planar magnetron as N-S-N and that of a circular planar magnetron as S-N-S while Vossen and Kern (1991) gave a S-N-S configuration for both circular and square planar magnetrons. Magnetic field strengths and polarity of the magnets in the US GUN II magnetrons supplied to our laboratory is not indicated in the operation manual as supplied by the manufacturer [US Patent No. 4,434,042, 1992].

Reproducibility in deposition rate problem in thin film deposition using magnetrons in our laboratory have forced us to study the magnetic field strength of new and damaged (used) magnetrons as well as changes in deposition rate with magnetron operation time. The study intends to investigate the magnetic field strength, magnet polarity of the magnetrons, and changes in deposition rate with magnetron operation time. We believe that these parameters have close associations to reproducibility problem in magnetron sputtering.

\section{EXPERIMENTAL}

Field strengths for four US Gun II magnetrons were used in this study, among which three (named, OLD1, OLD2, and OLD3) had been damaged and one (named NEW1) 
was brand new. Field strength for magnetron, NEW1 after it had been used and damaged (named NEW1d) was also investigated. Figure 1 shows the schematic diagram of the surface of the magnetron; distances used for measurement of magnetic field strength, B, are given.

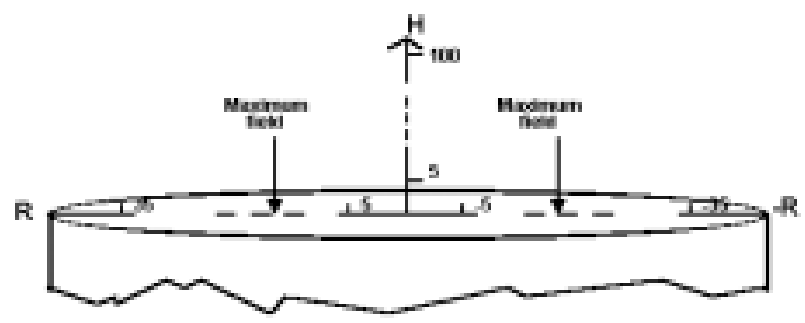

Figure 1. Schematic diagram of the surface of the magnetron. Distances, ( $\mathrm{R}$ to $-\mathrm{R}$ ) and 0 to $\mathrm{H}$ used for magnetic field strength measurements are in $\mathrm{mm}$

Transverse magnetic field was measured along the surface (at points $\mathrm{R}$ to $-\mathrm{R}$ ) as well as along perpendicular (at points 0 to $\mathrm{H}$ ) to the surface of the magnetron at two locations where the field was maximum along the surface. Care was taken to position the transverse probe of the gauss meter ( Lake Shore- Model 410 ) vertical to the surface of the magnetron. The orientation of the probe was positioned such that if the magnetic field lines are along $-R$ to $R$, it will give a positive value for the magnetic field strength.

Deposition rates for $\mathrm{Cu}$ and In targets with magnetron operation time (cumulative times during presputtering and sputtering) were monitored. These were obtained by dividing the film thickness by deposition time. $\mathrm{Cu}$ film thickness was obtained by using a Dektak while In film thickness was by weighing method. All depositions were carried out using a Balzers 250 coating unit at magnetron DC power of $200 \mathrm{~W}$.

\section{RESULTS AND DISCUSSION}

Magnetic field strength at the surface of the magnetron for different magnetrons is shown in Figure 2. Generally, magnetic field strength for all magnetrons changes sign with two maximas on both sides of $\mathrm{R}$ as the gauss meter probe traverses from $R=-35$ to $35 \mathrm{~mm}$. In this case the magnetic field lines change direction while the magnetic field strength changes from minimum to maximum and vice versa in a cycle. The change of direction is due to the surface of the magnetrons having either N-S-N or S$\mathrm{N}-\mathrm{S}$ magnetic poles configuration. Symmetry about the mid point of magnetrons is also observed.

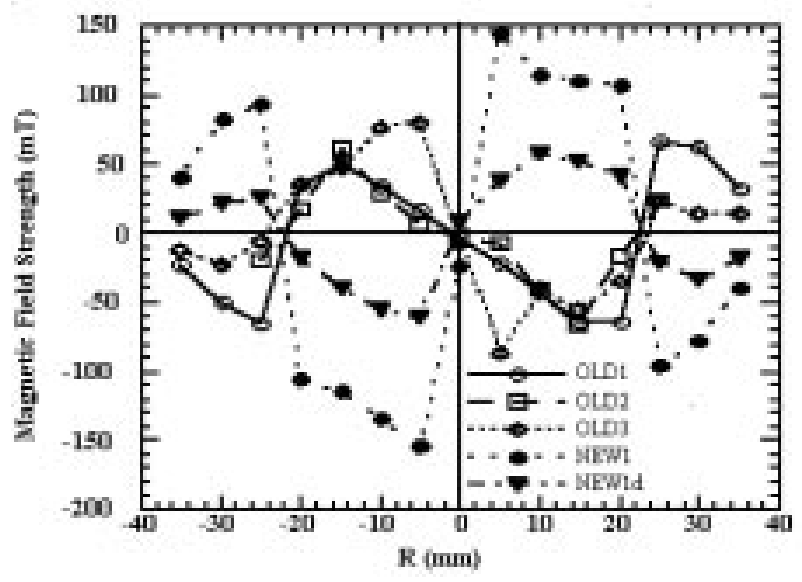

Figure 2. Magnetic field strengths at the surface for different magnetrons.

The maximum magnetic field strength is about -150 and $150 \mathrm{mT}$ for the new magnetron (NEW1) which occur at $\mathrm{R}=-5$ and $\mathrm{R}=5 \mathrm{~mm}$. For the used or old magnetrons, the maximum magnetic field strength is weaker than the new one by a factor of two to three. Polarity of the magnets for the old and the new magnetrons are different. The old magnetrons have a N-S-N configuration while the new one has a S-N-S configuration. This, however, does not mean that magnetic polarity changes when a magnetron is damaged;. Magnetron, NEW1 and NEWd exhibited the same polarity, S-N-S (see Figure 2).

Transverse magnetic field strength at distance ,H, perpendicular to the surface of the magnetrons is shown in Table I. Graphical representation for magnetrons NEW1 and NEWld is also shown in Fig. 3. Magnetic field strength reduces exponentially from a maximum value of about $150 \mathrm{mT}$ for the new magnetron, NEW1 and $60 \mathrm{mT}$ for the damaged magnetron, NEW1d to about $0 \mathrm{mT}$ at $\mathrm{H}$ U $40 \mathrm{~mm}$. Depending on the thickness of a target, the surface of the target on the magnetron can lie between $\mathrm{H}=3$ to $6 \mathrm{~mm}$. From Fig. 3 , the magnetic field strength is between about 10 to $40 \mathrm{mT}$ and 50 to $100 \mathrm{mT}$ for NEWd and NEW1 respectively at $\mathrm{H}=3$ to $6 \mathrm{~mm}$. Data for NEW1 agree very well with literature [Vossen and Kern, 1991; Thornton, 1978; Thornton and Penfold, 1978; Thornton, 1979]. 
TABLE I. Transverse magnetic field at distance, $\mathrm{H}$, from points at the surface of the magnetron.

\begin{tabular}{|l|l|l|l|l|l|l|l|l|l|l|}
\hline $\begin{array}{l}\mathrm{H} \\
(\mathrm{mm})\end{array}$ & $\begin{array}{l}\mathrm{B} \\
(\mathrm{mT})\end{array}$ & & & & & & & & & \\
\hline & OLD1 & & OLD2 & & OLD3 & & NEW1 & & NEWd \\
\hline & $\mathrm{R}=25$ & $\mathrm{R}=-25$ & $\mathrm{R}=15$ & $\mathrm{R}=-15$ & $\mathrm{R}=5$ & $\mathrm{R}=-5$ & $\mathrm{R}=5$ & $\mathrm{R}=-5$ & $\mathrm{R}=10$ & $\mathrm{R}=-5$ \\
\hline 0 & 66.10 & -65.00 & -66.50 & 61.60 & -81.30 & 80.45 & 143.25 & -154.75 & 58.7 & -61.10 \\
\hline 5.0 & 15.20 & -25.70 & -17.50 & 18.20 & -23.10 & 29.30 & 42.40 & -56.00 & 20.5 & -15.60 \\
\hline 10.0 & 7.12 & -11.36 & -8.56 & 9.84 & -7.34 & 8.35 & 14.65 & -17.70 & 10.99 & -7.17 \\
\hline 15.0 & 5.35 & -9.53 & -4.00 & 3.97 & -3.75 & 3.72 & 5.94 & -8.02 & 6.28 & -3.52 \\
\hline 20.0 & 4.27 & -5.81 & -2.00 & 0.72 & -1.99 & 1.07 & 3.30 & -2.53 & 3.06 & -1.02 \\
\hline 25.0 & 2.72 & -4.40 & -0.81 & -0.33 & -1.38 & 0.14 & 1.12 & -0.57 & 1.27 & 0.12 \\
\hline 30.0 & 2.01 & -3.67 & -0.57 & -0.65 & -0.81 & -0.26 & 0.10 & 0.19 & 0.54 & 0.45 \\
\hline 40.0 & 1.08 & -2.65 & -0.24 & -0.73 & -0.49 & -0.39 & -0.35 & 0.28 & 0.22 & 0.27 \\
\hline 50.0 & 0.51 & -1.82 & -0.21 & -0.61 & -0.41 & -0.40 & -0.42 & 0.12 & -0.28 & 0.04 \\
\hline 100.0 & -0.15 & -0.51 & -0.21 & -0.32 & -0.25 & -0.28 & -0.33 & -0.21 & -0.27 & -0.24 \\
\hline
\end{tabular}

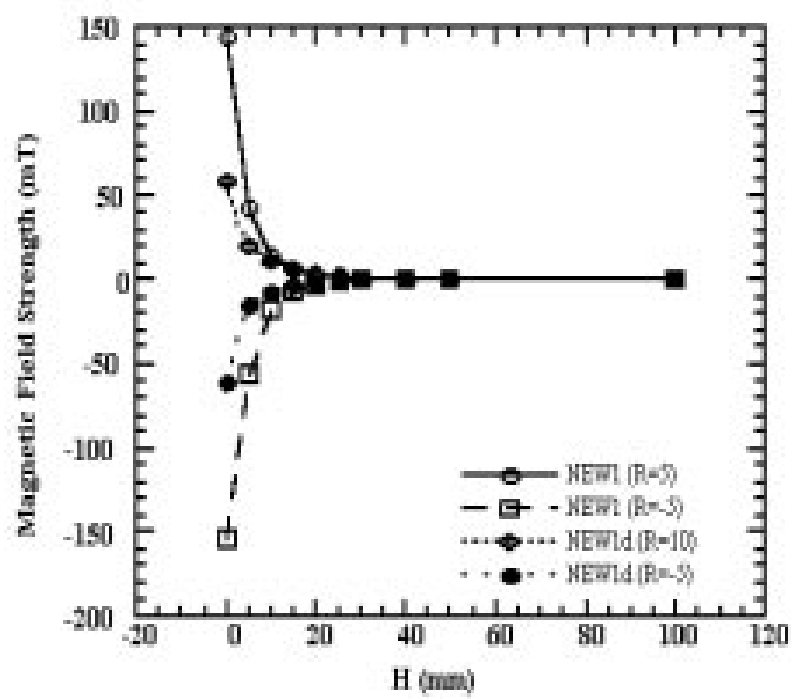

Figure 3. Transverse magnetic field strength for magnetrons NEW 1 and NEW 1d at perpendicular distance $H$ to the surface of the magnetron.

Figure 4 shows changes in deposition rate with magnetron operation time for $\mathrm{Cu}$ and $\mathrm{In}$ targets. The deposition rates were found to change from 4.84 to $4.63 \AA$ As- 1 (for $\mathrm{Cu}$ target) and 6.38 to $5.66 \AA$ As-1 (for In target) for cumulative magnetron operation times of about 14 and 13 hours respectively. Although the deposition rates changes appear to be within experimental errors (error in $\mathrm{Cu}$ and In deposition rates were $\pm 0.48 \AA s-1$ and $\pm 0.64 \AA$ As- 1 , respectively), graphically a trend where a gradual decrease in deposition rate with cumulative magnetron operation time is observed. However, a definitive trend needs further investigation. Installation of a new magnetron also had an effect on deposition rates inspite of keeping the deposition parameters constant. For two different magnetrons, data in our laboratory shows that for the first run ( denote as 0 operation time), deposition rates were 4.00 and 4.84 Ås-1 for $\mathrm{Cu}$ film and 6.38 and 1.18 Ås-1 for In film. The changes might be due to changes in target thickness, target erosion profile, and magnetic field strengths of magnetrons.

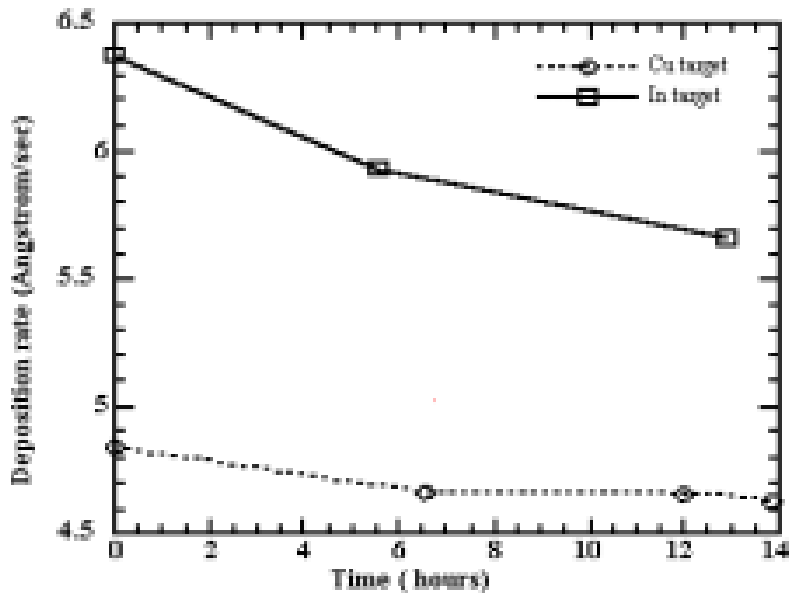

Figure 4. Deposition rate with magnetron operation time for $\mathrm{Cu}$ and $\mathrm{In}$ target.

\section{CONCLUSION}

Reproducibility problem in magnetron sputtering might be attributed to damaging of magnetrons and/or prolonged use of magnetrons. In addition to weakening of magnetic field strength, damaging of magnetrons demands installation of new magnetrons which can have different magnetic field strength or magnet polarity. These can change the deposition rate. However, a definitive conclusion requires a detailed investigation for the effect of magnetic polarity on deposition rate. For a magnetron to be in working condition, the transverse magnetic field strength on the surface of the magnetron should be about $50 \mathrm{mT}$ or more depending upon size and geometry of magnetron and target. Prolonged use of magnetrons results in weakening of magnetic field strength and in turn the need to install new magnetrons. Target surface erosion profile is also thought to affect the consistence of deposition rates. To avoid reproducibility problem in magnetron sputtering, frequent calibrations in deposition rate should be carried out with calibration being after installation of new target or magnetron and should repeat such calibrations as frequently as the need shall arise. Varying of deposition rate results in change of flux impinging onto the substrate which in turn causes modified growth process with changed film microstructures. 


\section{ACKNOWLEDGMENT}

J. M. M. thanks Deutscher Akademischer Austauschdienst (DAAD), Germany, through the African Network for Scientific and Technological Institutions (ANSTI) for providing scholarship. IPPS, Sweden, through the Solar Energy Group, University of Dar es Salaam, Tanzania, is thanked for providing research equipment and materials. Dr. G. Mbise is thanked for useful comments.

\section{REFERENCES}

U.S. Patent No. 4,434,042 (1992), US Gun II Owners Operation and Maintenance Manual of the US' Inc. sputtering source.

Vossen J. L. and Kern W. (1991), Thin Film Processes II (Academic press, New York), pp. 12-78

Thornton J. A. (1978), J. Vac. Sci. Technol. 15, p.171

Thornton J. A. and Penfold A. S. (1978), in "Thin Film Processes" (J.L. Vossen and W. Kern, eds.), (Academic Press, New York, 1978), p.75

Thornton J. A. (1979), Metal Finishing, 77, 45 (1979).

Vossen J. L. and Kern W. (1991), Thin Film Processes II (Academic press, New York, 1991), pp. 47-51, 184

Chapman B. (1980), Glow Discharge Processes, (John Wiley \& Sons, New York, 1980), pp. 267-268. 